\title{
INTEREST RATE MODELLING IN THE PRESENCE OF DISCONTINUITIES AND ITS SENSITIVITIES
}

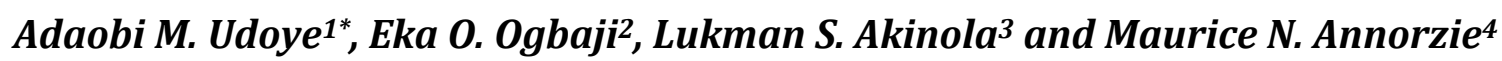

\begin{abstract}
${ }^{* 1,3}$ Department of Mathematics, Faculty of Science, Federal University Oye-Ekiti, Ekiti, Nigeria ${ }^{2}$ Department of Mathematics and Statistics, Faculty of Pure and Applied Sciences, Federal University Wukari, Taraba, Nigeria ${ }^{4}$ Department of Mathematics, Faculty of Physical Sciences, Imo State University, Owerri, Nigeria
\end{abstract}

Received 23rd December, 2020, Accepted 15 ${ }^{\text {th }}$ April, 2021

DOI: 10.2478/ast-2021-0002

*Corresponding author

Adaobi M. Udoye E-mail: adaobi.udoye@fuoye.edu.ng

Tel: $+234-8030866529$

\begin{abstract}
Interest rate paths experience discontinuities in the presence of certain factors. Much of the work on interest rate modelling has no consideration for effects of such unexpected occurrences in real life. A good risk manager needs to have a better model that considers possibility of unexpected occurrences. In this paper, we discuss step by step extension of Vasicek model to both jump model and jumpdiffusion model using Itô's formula as the major tool. We also derive the greeks 'delta' and 'vega' that measure sensitivity of the interest rate with respect to both changes in its initial interest rate and volatility in an interbank rate.
\end{abstract}

Keywords: Interest Rate, Vasicek model, Compound Poisson process, Jump-diffusion process, Itô formula

MSC2020: 60J75, 60J60, 91G15 


\subsection{Introduction}

A good model should consider behaviours inherent in the system under consideration. A given system behaves abnormally because of certain circumstances. Factors such as change in government policies, inflation, natural disasters, presence of pandemic (e.g. corona virus), etc contribute to spikes in the interest rates. To solve the problem of getting a better model that takes care of discontinuities in options, Merton (1976) obtained a pricing formula for underlying stock returns produced by both continuous and discontinuous (jump) processes. He further highlighted the extension of the formula to the pricing of corporate liabilities. Kou (2002) derived a double exponential jumpdiffusion model for the valuation of options and emphasised on the importance of jump-diffusion model for pricing assets in financial engineering. Later, many researchers have contributed to the relevance of the jump-diffusion model in different areas. Buraschi and Jiltsov (2007) observed that interest rates exhibit continuous and discontinuous behaviours, as a result, they suggested obtaining solutions of term structure model under jump-diffusion processes. Berhane et al. (2019) derived a better model for Ethiopian commodity prices to minimise risks caused by jumps using the jump-diffusion model. Carr and Mayo (2007) proposed numerical pricing of options with partial integral differential equation when density functions of jump processes have polynomials, Gaussian and exponential. Feng and Linetsky (2008) discussed a computational technique involving partial integro differential equation for options' pricing in jump-diffusion model. Bates (1996) proposed an approach for stochastic volatility model driven by the model. Chang and Wang (2020) presented option pricing driven by double stochastic volatility model in conjunction with stochastic interest rate model and double exponential jumps and stochastic intensity. Lau et al. (2019) presented an approach to estimate market parameters modelled using a certain jump-diffusion model. Novat et al. (2019) discussed Merton's jump-diffusion model in stock markets of three East African countries. Salmi and Toivanen (2011) derived iterative steps for valuing American option using the model. Wang et al. (2017) proposed pricing of vulnerable American option under jump-diffusion process, whereas Jiahui et al. (2019) obtained a closed form evaluation formula for that of European option having credit and jump risks under inadequate information. Perelló et al. (2020) focused on stochastic interest rate modelling for assessing future with ramification in climate change, while Verschuren (2020) developed a coherent structure on how to include certain interest rates in a single curve stochastic term structure model and did comparison with multiple curve analogue.
In this paper, we shall employ the jump-diffusion model in the modelling of interest rate consisting of an interbank offered rate by modifying Vasicek short rate model and obtain expression for the greeks 'delta' and 'vega' of the interest rate using integration by parts technique of Malliavin calculus as given by Bavouzet-Morel and Messaoud (2006). Wu and Liang (2018) studied the Vasicek model with mixed-exponential jumps and its applications in finance and insurance, by first obtaining the explicit form of Laplace transform of the model distribution through the aid of piecewise deterministic Markov process theory and the martingale theory. Rao (2021) discussed asymptotic properties of the maximum likelihood estimator of unknown parameters in a fractional Vasicek model driven by a fractional Brownian motion, while Guo (2021) evaluated the benefit of bias-correction method in enhancing the Vasicek model for market risk management. In the next section, we discuss the important mathematical tools needed for the success of the paper.

\subsection{Experimental}

In this section, we discuss important mathematical tools required for the success of the work.

The Vasicek model (1977): The model has the property of meanreversion and interest rates are known to revert back to certain mean after sometime. Hence, it is necessary that a good model of interest rates should be able to take care of its mean-reversion feature. The dynamics for the Vasicek short rate model is given by

$$
d r_{t}=\alpha\left(\rho-r_{t}\right) d t+\sigma d W_{t},
$$

where $r_{t}$ is the interate rate at time $t ; \alpha, \rho$ and $\sigma$ represent speed of mean-reversion, long-term mean rate and volatility of the interest rate, respectively, while $W_{t}$ denotes the Wiener process.

Itô's formula for jump-diffusion process (Cont and Tankov, 2004): Let $Y=Y_{t}$ be a diffusion process with jumps at time $t$ defined as

$$
Y_{t}=Y_{0}+\int_{0}^{t} a_{s} d s+\int_{0}^{t} \sigma_{s} d W_{s}+\sum_{i=1}^{N_{t}} \Delta Y_{i}
$$

where $a_{s}$ and $\sigma_{s}$ are non-anticipating processes with $E\left[\int_{0}^{t} \sigma_{t}^{2} d t\right]<\infty$, respectively, and $0 \leq s \leq t$.

$$
\begin{aligned}
& f\left(t, Y_{t}\right)=f\left(0, Y_{0}\right)+\int_{0}^{t}\left[\frac{\partial f\left(s, Y_{s}\right)}{\partial s}+a_{s} \frac{\partial f\left(s, Y_{s}\right)}{\partial y}+\frac{1}{2} \sigma_{s}^{2} \frac{\partial^{2} f\left(s, Y_{s}\right)}{\partial y^{2}}\right] d s \\
& +\int_{0}^{t} \frac{\partial f\left(s, Y_{s}\right)}{\partial y} d W_{s}+\sum_{i \geq 1, T_{i} \leq t}\left[f\left(Y_{T_{i-}}+\Delta Y_{i}\right)-f\left(Y_{T_{i_{-}}}\right)\right] .
\end{aligned}
$$

Then, for each $C^{1,2}$ function $f:[0, T] \rightarrow R$, the process $f\left(t, Y_{t}\right)$ can be written as

Itô's formula for jump process

Suppose that $X_{t}$ is a jump process having values in $R$ given as

$$
Y_{t}=\int_{0}^{t} a_{s} d s+\sum_{i=1}^{N_{t}} \Delta Y_{i}
$$


where $a_{s}$ is a non-anticipating left continuous with right limit process, $\Delta Y_{i}=Y\left(T_{i}\right)-Y\left(T_{i_{-}}\right)$denote the $i$ th jump size and $N_{t}$ represents the random number of jumps between 0 and $t$. Let $\left(T_{n}\right)_{n \geq 1}$ be the jump times of $Y_{t}$, then the following holds:

$$
\begin{gathered}
f\left(Y_{T}\right)=f\left(Y_{0}\right)+\int_{0}^{T} a_{t} \frac{\partial f\left(Y_{t}\right)}{\partial y} d t+\sum_{\left\{i, T_{i} \leq t\right\}} f\left(Y_{T_{i_{-}}}+\Delta Y_{t}\right)-f\left(y_{T_{i_{-}}}\right) \\
=f\left(Y_{0}\right)+\int_{0}^{T} a_{t^{-}} \frac{\partial f\left(Y_{t^{-}}\right)}{\partial y} d t \\
\quad+\sum_{0 \leq t \leq T}^{\Delta X_{t} \neq 0}\left[f\left(Y_{T_{i_{-}}}+\Delta Y_{t}\right)-f\left(X_{t_{-}}\right)\right]
\end{gathered}
$$

where the sum covers the random jumps.

\section{The Malliavin Calculus}

Let $(\Omega, \mathfrak{F}, \mathbb{P})$ be a given probability space where $\Omega, \mathfrak{F}$ and $\mathbb{P}$ denote sample space, set of filtration and the probability measure. Let $\left(G_{n}\right)_{n \in N}$ be a sequence of independent random variables on $(\Omega, \mathfrak{F}, \mathbb{P})$ with density function $\breve{f}_{n}$ and assume that $G_{n}$ has moments of any order for $n \geq 1$ such that $G_{n}$ is absolutely continuous and continuously differentiable in $R$ with $\lim _{n \rightarrow \infty}|y|^{n} \breve{f}_{n}(y)=0$ and let $\partial_{y} \ln \left(\breve{f}_{n}(y)\right)=$ $\frac{\partial_{y} \breve{f}_{n}(y)}{\breve{f}_{n}(y)}$ has at most polynomial growth. $f \in C^{k}\left(R^{n}\right)$ represents the function $f: R^{n} \rightarrow R$ that are $k$ times continuously differentiable with derivatives of $f$ and $f$ permitting at most polynomial growth. Following Bavouzet-Morel and Messaoud (2006), we have the definitions:

i. A random variable $F=f\left(G_{1}, \ldots, G_{n}\right)$ where $f: R^{n} \rightarrow R$ is a measurable function for some $n \in N$, is called a simple functional. $S_{(n, k)}$ denotes the space of simple functional.

ii. A sequence of random variables $U=\left(U_{i}, i \leq n\right)$ is called a simple process of length nprovided $U_{i}=$ $u_{i}\left(G_{1}(w), \ldots, G_{n}(w)\right)$. The space of simple processes of length $n$ is denoted by $P_{(n, k)}$.

iii. The Malliavin derivative is defined as $D: S_{(n, 1)} \rightarrow P_{(n, 0)}$ such that if $F=f\left(G_{1}, \ldots, G_{n}\right)$ then

$$
D_{i} F=\left(\frac{\partial f}{\partial x_{i}}\right)\left(G_{1}(w), \ldots, G_{n}(w)\right) \text {. }
$$

iv. The Skorohod integral operator defined by $\delta: P_{(n, 1)} \rightarrow S_{(n, 0)}$ is such that

$$
\delta(U)=-\sum_{i=1}^{n}\left(D_{i} U_{i}+\varphi_{i}\left(G_{i}\right) U_{i}\right)
$$

where $\varphi_{i}(y)=\frac{\partial \ln \breve{f}_{i}(y)}{\partial y}$ if $\breve{f}_{i}(y)>0$; otherwise, it is equal to 0 .

v. The Ornstein-Uhlenbeck operator defined as $L: S_{(n, 2)} \rightarrow S_{(n, 0)}$ is a Malliavin derivative operator on the Skorohod integral, that is,

$L F=-\sum_{i=1}^{n}\left[D_{i} D_{i} F+\varphi_{i} D_{i} F\right], \quad i=1, \ldots, n$.

vi. Let $F=\left(F^{1}, \ldots, F^{d}\right), F^{i} \in S_{(n, 1)}$. Then, the Malliavin covariance matrix is defined as

$$
M^{i j}(F)=\left\langle D F^{i}, D F^{i}\right\rangle
$$

$$
=\sum_{p=1}^{n}\left(\partial_{p} f^{i} \partial_{p} f^{j}\right)\left(G_{1}, \ldots, G_{n}\right) .
$$

The Malliavin integration by parts theorem. Suppose that $F=$ $\left(F^{1}, \ldots, F^{d}\right) \in S_{(n, 2)}^{d}, \quad B \in S_{(n, 1)}$. Let $M(F)$ be an invertible matrix with its inverse denoted by $M^{-1}(F)$. Let $E\left[\left(\operatorname{det} M^{-1}(F)\right)^{4}\right]<\infty$. Then, for any smooth function $\Phi: R^{d} \rightarrow R$, any $i=1, \ldots, d$,

$$
E\left[\partial_{i} \Phi(F) B\right]=E\left[\Phi(F) H^{i}(F, B)\right]
$$

where

$$
\begin{aligned}
& H^{i}(F, B) \\
= & \sum_{i=1}^{d} B\left(M^{-1}\right)^{j i}(F) L F^{j}-\left(M^{-1}\right)^{j i}(F)\langle D F, D B\rangle \\
- & B\left\langle D F^{j}, D\left(D M^{-1}\right)^{j i}(F)\right\rangle .
\end{aligned}
$$

\subsection{Results and Discussion}

In this section, we derive the modified Vasicek model driven by pure jump and jump-diffusion processes to settle different scenarios with the aid of Bavouzet-Morel and Messaoud (2006). The interest rate paths experience spikes as can be seen in figure 1 showing Nigerian interbank offered rates data between January 2007 and July 2020 obtained from the website of the Central Bank of Nigeria.

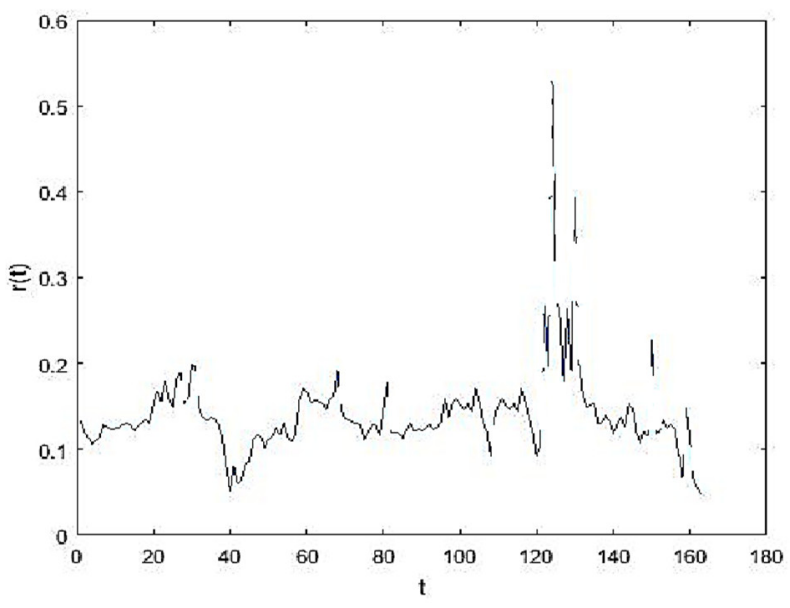

Fig. 1: Interest rate paths.

Theorem 3.1. Let $r_{t}$ be an interest rate at time and let the short rate dynamics of the interest rate be given by

$$
d r_{t}=\alpha\left(\rho-r_{t}\right) d t+\sigma d W_{t}+d J_{t}
$$

where $\alpha, \rho, \sigma$ and $W_{t}$ represent speed of mean-reversion, long-term mean rate, volatility and Wiener process of the interest rate, respectively, while $J_{t}$ denotes compound Poisson process that can be written as

$$
J_{t}=\sum_{i=1}^{N_{t}} \Delta r_{i}
$$

where $N_{t}$ is a Poisson process that counts the number of jumps to time $t$ and $\Delta r_{i}=r_{T_{i}}-r_{T_{i-1}}$ represents jump sizes that occur at jump times ' $T_{i}$ '. Then, $r_{t}=r(t)$ is given by

$$
\begin{gathered}
r_{t}=r_{0} e^{-\alpha t}+\rho\left(1-e^{-\alpha t}\right)+\sigma \int_{0}^{t} e^{-\alpha(t-s)} d W_{s} \\
+\sum_{i=1}^{n} \Delta r_{i} e^{-\alpha\left(t-T_{i}\right)} .
\end{gathered}
$$

Proof. Using Itô's formula, let $f=r_{t} e^{\alpha t}, \frac{\partial f}{\partial s}=\alpha r e^{\alpha t}, \frac{\partial f}{\partial r}=e^{\alpha t}$ and $\frac{\partial^{2} f}{\partial r^{2}}=0$. Then,

$$
r_{t} e^{\alpha t}=r_{0}+\int_{0}^{t}\left(\alpha r e^{\alpha s}+(\alpha(\rho-r)) e^{\alpha s}\right) d s+\int_{0}^{t} \sigma e^{\alpha s} d W_{s}
$$


Whence,

$$
\begin{gathered}
+\sum_{i \geq 1, T_{i} \leq t}\left[r_{t} e^{\alpha t}+\Delta r_{i} e^{\alpha T_{i}}-r_{t} e^{\alpha t}\right] \\
=r_{0}+\int_{0}^{t} \alpha \rho e^{\alpha s} d s+\int_{0}^{t} \sigma e^{\alpha t} d W_{s}+\sum_{\substack{i \geq 1, T_{i} \leq t \\
N_{t}}} \Delta r_{i} e^{\alpha T_{i}} \\
=r_{0}+\rho\left(1-e^{-\alpha t}\right)+\sigma \int_{0}^{t} e^{\alpha t} d W_{s}+\sum_{i=1}^{\Delta r_{i} e^{\alpha T_{i}}}
\end{gathered}
$$

$$
r_{t}=r_{0} e^{-\alpha t}+\rho\left(1-e^{-\alpha t}\right)+\sigma \int_{0}^{t} e^{-\alpha(t-s)} d W_{s}+\sum_{i=1}^{N_{t}} \Delta r_{i} e^{-\alpha\left(t-T_{i}\right)} .
$$

Hence, equation (3.3) is obtained by substituting $n=N_{t}$.

Remark. If there is no jump, the original Vasicek model can be recovered as shown in the corollary below.

Corollary 3.2. Let $r_{t}$ be a jump-diffusion interest rate model with zero jumps. Then,

$$
r_{t}=r_{0} e^{-\alpha t}+\rho\left(1-e^{-\alpha t}\right)+\int_{0}^{t} e^{-\alpha(t-s)} d W_{s}
$$

which can be written as

$$
r_{t}=r_{0} e^{-\alpha t}+\rho\left(1-e^{-\alpha t}\right)+\frac{\sigma^{2}}{2 \alpha}\left(1-e^{-2 \alpha t}\right) t .
$$

Proof. From equation (3.3), we have that $N_{t}=0$ which implies that the jump sum is equal to zero whenever there is no jump. Thus, it follows that equation (3.4) is valid.

Furthermore, the expectation of $r_{t}$ in equation (3.4) is given by

while the variance is given by

$$
E\left[r_{t}\right]=r_{0} e^{-\alpha t}+\rho\left(1-e^{-\alpha t}\right)
$$

$$
\begin{gathered}
\operatorname{Var}\left[r_{t}\right]=E\left[r_{t}-E\left[r_{t}\right]\right]^{2}=E\left[\sigma^{2} e^{-2 a t} \int_{0}^{t} e^{2 \alpha s} d s\right] \\
=\frac{\sigma^{2}}{2 \alpha}\left(1-e^{-2 \alpha}\right) .
\end{gathered}
$$

Summing the expectation and variance of the interest rate $r_{t}$, we obtain equation (3.5).

Theorem 3.3. The extended Vasicek model driven by pure jump process is given by the dynamics

$$
d r_{t}=\alpha\left(\rho-r_{t}\right) d t+\sigma d J_{t}
$$

and its solution is given by

$$
r_{t}=r_{0} e^{-\alpha t}+\rho\left(1-e^{-\alpha t}\right)+\sigma \sum_{i=1}^{n} \Delta r_{i} e^{-\alpha\left(t-T_{i}\right)} .
$$

Proof. Using Itô's formula in Section 2.3, we let $f\left(r_{t}\right)=r_{t} e^{\alpha t}$ and $\frac{\partial f}{\partial r}=$ $e^{\alpha t}$. Thus,

$$
\begin{gathered}
r_{t} e^{\alpha t}=r_{0}+\int_{0}^{t}\left(\alpha r e^{\alpha s}+\alpha\left(\rho-r_{s}\right) e^{\alpha s}\right) d s \\
+\sigma \sum_{i \geq 1, T_{i} \leq t}\left[r_{t} e^{\alpha t}+\Delta r_{i} e^{\alpha T_{i}}-r_{t} e^{\alpha t}\right] \\
=r_{0}+\rho\left(e^{\alpha t}-1\right)+\sigma \sum_{i \geq 1, T_{i} \leq t}\left[r_{t} e^{\alpha t}+\Delta r_{i} e^{\alpha T_{i}}-r_{t} e^{\alpha t}\right] \\
=r_{0}+\rho\left(e^{\alpha t}-1\right)+\sigma \sum_{i=1}^{N_{t}} \Delta r_{i} e^{\alpha T_{i}} \\
r_{t}=r_{0} e^{-\alpha t}+\rho\left(1-e^{-\alpha t}\right)+\sigma \sum_{i=1}^{N_{t}} \Delta r_{i} e^{\alpha\left(T_{i}-t\right)} .
\end{gathered}
$$

\section{Sensitivities computation}

In the following, $E[\cdot]$ denotes expected payoff function, $\Phi\left(r_{T}\right)=$ $\max \left(r_{T}-K, 0\right)$ is the payoff function of the interest rate, $K$ is the strike price and $T$ is the maturity time.

European call price on $r_{T}$ is given by

$$
V=e^{-r_{0} T} E\left[\Phi\left(r_{T}\right)\right]
$$

Furthermore $F=r_{T}$.

From equation (3.3), we adopt the interest rate given by

$$
\begin{gathered}
r_{T}=r_{0} e^{-\alpha T}+\rho\left(1-e^{-\alpha T}\right)+\sigma \sum_{k=1}^{m} e^{-\alpha\left(T-t_{k}\right)} \sqrt{t_{k}-t_{k-1}} Z_{k} \\
+\sum_{i=1}^{n} \Delta r_{i} e^{-\alpha\left(T-T_{i}\right)}
\end{gathered}
$$

where

$$
W\left(t_{k}\right)-W\left(t_{k-1}\right)=\sqrt{t_{k}-t_{k-1}} Z_{k} .
$$

The Malliavin derivative on $r_{T}$ is given by

$$
D F=D r_{T}=D r_{T}^{W}+D r_{T}^{\Delta_{i}} \text {. }
$$

We obtain $D r_{T}^{W}$ as

$$
=\sigma \sum_{k=1}^{m} e^{-\alpha\left(T-t_{k}\right)} \sqrt{t_{k}-t_{k-1}} .
$$

We obtain $D r_{T}^{\Delta_{i}}$ as

$$
D r_{T}^{\Delta_{i}}=\sigma \sum_{i=1}^{n} e^{-\alpha\left(T-T_{i}\right)} .
$$

The Malliavin covariance matrix on $r_{T}$ is given by

$$
M(F)=M^{W}(F)+M^{\Delta_{i}}(F)
$$

where

$$
M^{W}(F)=\sigma^{2} \sum_{k=1}^{m} e^{-2 \alpha\left(T-t_{k}\right)}\left(t_{k}-t_{k-1}\right)
$$

and

$$
M^{\Delta_{i}}(F)=\sum_{i=1}^{n} e^{-2 \alpha\left(T-T_{i}\right)} .
$$

The inverse Malliavin covariance matrix is given by

$$
M^{-1}(F)=M^{-1}\left(r_{T}\right)=M_{W}^{-1}\left(r_{T}\right)+M_{\Delta_{i}}^{-1}\left(r_{T}\right) .
$$

From equations (3.13) and (3.14), we have

$$
M_{W}^{-1}\left(r_{T}\right)=\frac{1}{\sigma^{2}} \sum_{k=1}^{m} \frac{e^{2 \alpha\left(T-t_{k}\right)}}{t_{k}-t_{k-1}}
$$

and

$$
M_{\Delta_{i}}^{-1}\left(r_{T}\right)=\sum_{i=1}^{n} e^{2 \alpha\left(T-T_{i}\right)} .
$$

The Malliavin derivative on the inverse Malliavin covariance matrix is given by

$$
D M_{W}^{-1}=0=D M_{\Delta_{i}}^{-1}
$$

The Ornstein-Uhlenbeck operator on $r_{T}$ is given by

$$
L F=L r_{T}=-\left[D D r_{T}+\varphi D r_{T}\right]=L r_{T}^{W}+L r_{T}^{\Delta_{i}} \text {. }
$$

From equations (3.8) and (3.15), the Malliavin derivative $D D r_{T}=0$ since

$$
\begin{gathered}
D D r_{T}^{W}=0=D D r_{T}^{\Delta_{i}} . \\
\varphi=\frac{\partial \ln \breve{f}(z)}{\partial z}=\frac{\partial \ln \left(\frac{1}{\sqrt{2 \pi}} e^{-\frac{1}{2} z^{2}}\right)}{\partial z}=-z .
\end{gathered}
$$

Thus,

$$
=\sigma \sum_{k=1}^{m} e^{-\alpha\left(T-t_{k}\right)} \sqrt{t_{k}-t_{k-1}^{W}} Z_{k}
$$

and 


$$
=\sum_{i=1}^{n} e^{-\alpha\left(T-T_{T}\right)} \Delta_{i}^{\Delta_{i}}
$$

\section{Derivation of the greek 'delta' of the Interest Rate}

The greek 'delta' measures the sensitivity of the interest rate with respect to change in its initial value. It is given by

$$
\begin{gathered}
\text { delta }=\Delta=\frac{\partial V}{\partial r_{0}}=\frac{\partial}{\partial r_{0}} e^{-r_{0} T} E\left[\Phi\left(r_{T}\right)\right] \\
=-T e^{-r_{0} T} E\left[\Phi\left(r_{T}\right)\right]+e^{-r_{0} T} E\left[\Phi^{\prime}\left(r_{T}\right) \frac{\partial r_{T}}{\partial r_{0}}\right] \\
=-T e^{-r_{0} T} E\left[\Phi\left(r_{T}\right)\right]+e^{-r_{0} T} E\left[\Phi\left(r_{T}\right) H\left(r_{T}, \frac{\partial r_{T}}{\partial r_{0}}\right)\right] \\
=e^{-r_{0} T}\left(-T E\left[\Phi\left(r_{T}\right)\right]+E\left[\Phi\left(r_{T}\right) H\left(r_{T}, B_{r_{0}}\right)\right]\right)
\end{gathered}
$$

where

$$
B=B_{r_{0}}=\frac{\partial r_{T}}{\partial r_{0}}=e^{-\alpha T}
$$

and

$$
\begin{aligned}
H\left(r_{T}, B_{r_{0}}\right)=H(F, B) & =B M^{-1}(F) L F-B\left\langle D F, D M^{-1}(F)\right\rangle \\
& -M^{-1}\langle D F, D G\rangle
\end{aligned}
$$

is the Malliavin weight.

We proceed to obtain the Malliavin weight.

Malliavin derivative on $B=B_{r_{0}}$ satisfies

$$
D B=D B_{r_{0}}=0 \text {. }
$$

Theorem 3.4. Let the interest rate $r_{T}=r(T)$ be as given above, then the Malliavin weight for the greek 'delta' is given by

$$
\begin{aligned}
H(F, B)=\frac{e^{-\alpha T}}{\sigma} \sum_{k=1}^{m} \frac{e^{\alpha\left(T-t_{k}\right)}}{\sqrt{t_{k}-t_{k-1}}} Z_{k} \\
\\
\quad+e^{-\alpha T} \sum_{i=1}^{n} e^{\alpha\left(T-T_{i}\right)} \Delta_{i} .
\end{aligned}
$$

Proof.

The Malliavin weight

$$
H(F, B)=H^{W}(F, B)+H^{\Delta_{i}}(F, B) .
$$

We proceed to obtain $H^{W}(F, B)$.

Thus,

$$
H^{W}(F, B)=B M^{-1}(F) L F-M^{-1}(F)\langle D F, D B\rangle-B\left\langle D M^{-1}(F), D F\right\rangle .
$$

From equations (3.17) and (3.25), $D M^{-1}=0$ and $D B=0$, respectively. This implies that

$$
M^{-1}(F)\langle D F, D B\rangle=B\left\langle D M^{-1}(F), D F\right\rangle=0 .
$$

Thus, from equations (3.24), (3.12) and (3.22), we obtain $H^{W}(F, B)=B M^{-1}(F) L F$

$$
\begin{aligned}
& =e^{-\alpha T} \\
& \frac{1}{\sigma^{2}} \sum_{k=1}^{m} \frac{e^{2 \alpha\left(T-t_{k}\right)}}{t_{k}-t_{k-1}} \cdot \sigma e^{-\alpha\left(T-t_{k}\right)} \sqrt{t_{k}-t_{k-1}} Z_{k} \\
\therefore H^{W}(F, B) & =\frac{e^{-\alpha T}}{\sigma} \sum_{k=1}^{m} \frac{e^{\alpha\left(T-t_{k}\right)}}{\sqrt{t_{k}-t_{k-1}}} Z_{k} .
\end{aligned}
$$

We now derive $H^{\Delta_{i}}(F, B)$,

$$
H(F, B)=B M^{-1}(F) L F-M^{-1}(F)\langle D F, D B\rangle-B\left\langle D M^{-1}(F), D F\right\rangle .
$$

But from equations (3.17) and (3.25),

This implies that

$$
D M^{-1}=0=D B \text {. }
$$

$$
\begin{gathered}
H^{\Delta_{i}}(F, B)=B M^{-1}(F) L F=e^{-\alpha T} \cdot \frac{1}{\sigma^{2}} \sum_{i=1}^{n} e^{2 \alpha\left(T-T_{i}\right)} \cdot e^{\alpha\left(T-T_{i}\right)} \Delta_{i} \\
\therefore H^{\Delta_{i}}(F, B)=e^{-\alpha T} \sum_{i=1}^{n} e^{\alpha\left(T-T_{i}\right)} \Delta_{i} .
\end{gathered}
$$

From equations (3.20) and (3.21), the Malliavin weight with respect to the greek 'delta' becomes

$$
\begin{aligned}
H(F, B)=H^{W}(F, B) & +H^{\Delta_{i}}(F, B) \\
& =\frac{e^{-\alpha T}}{\sigma} \sum_{k=1}^{m} \frac{e^{\alpha\left(T-t_{k}\right)}}{\sqrt{t_{k}-t_{k-1}}} Z_{k}+e^{-\alpha T} \sum_{i=1}^{n} e^{\alpha\left(T-T_{i}\right)} \Delta_{i},
\end{aligned}
$$

Hence, the result follows.

\section{Derivation of the greek 'vega' of the Interest Rate}

The greek 'vega' measures the sensitivity of the interest rate with respect to change in its volatility. It is given by

$$
\begin{aligned}
& \quad \text { vega }=\vartheta=\frac{\partial V}{\partial \sigma}=\frac{\partial}{\partial \sigma} e^{-r_{0} T} E\left[\Phi\left(r_{T}\right)\right] \\
= & e^{-r_{0} T} E\left[\Phi\left(r_{T}\right) H\left(r_{T}, \frac{\partial r_{T}}{\partial \sigma}\right)\right] \\
= & e^{-r_{0} T} E\left[\Phi\left(r_{T}\right) H\left(r_{T}, B_{\sigma}\right)\right]
\end{aligned}
$$

where $H\left(r_{T}, B_{\sigma}\right)$ is the Malliavin weight with respect to the greek 'vega'.

From equation (3.9),

$$
\begin{gathered}
B_{\sigma}=\frac{\partial r_{T}}{\partial \sigma} \\
=\sum_{k=1}^{m} e^{-\alpha\left(T-t_{k}\right)} \sqrt{t_{k}-t_{k-1}} Z_{k} .
\end{gathered}
$$

The Malliavin derivative of $B_{\sigma}$ gives

$$
=\sum_{k=1}^{m} e^{-\alpha\left(T-t_{k}\right)} \sqrt{t_{k}-t_{k-1}} .
$$

Theorem 3.5. Let $r_{T}$ be given by equation (3.9). Then, the Malliavin weight for the greek 'vega' is given by

$$
\begin{aligned}
H\left(F, B_{\sigma}\right)=\frac{1}{\sigma} \sum_{k=1}^{m}\left(Z_{k}\right. & -1) \\
& +\sum_{k=1}^{m} e^{-\alpha\left(T-t_{k}\right)} \sqrt{t_{k}-t_{k-1}}\left(Z_{k} \sum_{i=1}^{n} e^{\alpha\left(T-T_{i}\right)} \Delta_{i}\right. \\
& \left.-\sum_{i=1}^{n} e^{\alpha\left(T-T_{i}\right)}\right) .
\end{aligned}
$$

Proof. The Malliavin weight is defined as

$$
\begin{gathered}
H\left(F, B_{\sigma}\right)=B_{\sigma} M^{-1}(F) L F-M^{-1}(F)\left\langle D F, D B_{\sigma}\right\rangle-B_{\sigma}\left\langle D M^{-1}(F), D F\right\rangle \\
=H^{W}\left(F, B_{\sigma}\right)+H^{\Delta_{i}}\left(F, B_{\sigma}\right) . \\
H(F, B)=H\left(F, B_{\sigma}\right)=B_{\sigma} M^{-1}(F) L F-M^{-1}(F)\left\langle D F, D B_{\sigma}\right\rangle \\
-B_{\sigma}\left\langle D M^{-1}(F), D F\right\rangle .
\end{gathered}
$$

From equation (3.17),

$$
D M^{-1}(F)=D M^{-1}\left(r_{T}\right)=0 .
$$

Thus,

$$
\begin{gathered}
H^{W}\left(F, B_{\sigma}\right)=B_{\sigma} M^{-1}(F) L F-M^{-1}(F)\left\langle D F, D B_{\sigma}\right\rangle-B_{\sigma}\left\langle D M^{-1}(F), D F\right\rangle \\
=B_{\sigma} M^{-1}(F) L F-M^{-1}(F)\left\langle D F, D B_{\sigma}\right\rangle .
\end{gathered}
$$

From equations (3.16), (3.15), (3.22), (3.10) and (3.29), we obtain

$$
\begin{aligned}
& H^{W}\left(F, B_{\sigma}\right)=\sum_{k=1}^{m} e^{-\alpha\left(T-t_{k}\right)} \sqrt{t_{k}-t_{k-1}} Z_{k} \\
& \cdot \frac{1}{\sigma^{2}} \sum_{k=1}^{m} \frac{e^{2 \alpha\left(T-t_{k}\right)}}{t_{k}-t_{k-1}} \cdot \sigma \sum_{k=1}^{m} e^{-\alpha\left(T-t_{k}\right)} \sqrt{t_{k}-t_{k-1}} Z_{k} \\
& -\frac{1}{\sigma^{2}} \sum_{k=1}^{m} \frac{e^{2 \alpha\left(T-t_{k}\right)}}{t_{k}-t_{k-1}} \cdot \sigma \sum_{k=1}^{m} e^{-\alpha\left(T-t_{k}\right)} \sqrt{t_{k}-t_{k-1}} \cdot \sum_{k=1}^{m} e^{-\alpha\left(T-t_{k}\right)} \sqrt{t_{k}-t_{k-1}} .
\end{aligned}
$$

Hence,

$$
H^{W}\left(F, B_{\sigma}\right)=\sum_{k=1}^{m} \frac{1}{\sigma} Z_{k}^{2}-\frac{1}{\sigma}=\frac{1}{\sigma} \sum_{k=1}^{m}\left(Z_{k}^{2}-1\right) .
$$


Furthermore, we have for the jump part,

$$
\begin{gathered}
H^{\Delta_{i}}\left(F, B_{\sigma}\right)=B_{\sigma} M^{-1}(F) L F-M^{-1}(F)\left\langle D F, D B_{\sigma}\right\rangle-B_{\sigma}\left\langle D M^{-1}(F), D F\right\rangle \\
=B_{\sigma} M^{-1}(F) L F-M^{-1}(F)\left\langle D F, D B_{\sigma}\right\rangle .
\end{gathered}
$$

From equations (3.24), (3.14), (3.17), (3.10) and (3.29), we get

$$
\begin{gathered}
H^{\Delta_{i}}\left(F, B_{\sigma}\right)=\sum_{k=1}^{m} e^{-\alpha\left(T-t_{k}\right)} \sqrt{t_{k}-t_{k-1}} Z_{k} \cdot \sum_{i=1}^{n} e^{2 \alpha\left(T-T_{i}\right)} \cdot e^{-\alpha\left(T-T_{i}\right)} \Delta_{i} \\
-\sum_{i=1}^{n} e^{2 \alpha\left(T-T_{i}\right)} \cdot e^{-\alpha\left(T-T_{i}\right)} \cdot \sum_{k=1}^{m} e^{-\alpha\left(T-t_{k}\right)} \sqrt{t_{k}-t_{k-1}} \\
=\sum_{k=1}^{m} e^{-\alpha\left(T-t_{k}\right)} \sqrt{t_{k}-t_{k-1}} Z_{k} \sum_{i=1}^{n} e^{\alpha\left(T-T_{i}\right)} \Delta_{i} \\
-\sum_{i=1}^{n} e^{\alpha\left(T-T_{i}\right)} \sum_{k=1}^{m} e^{-\alpha\left(T-t_{k}\right)} \sqrt{t_{k}-t_{k-1}} .
\end{gathered}
$$

Thus,

$$
\begin{gathered}
H^{\Delta_{i}}\left(F, B_{\sigma}\right)=\sum_{k=1}^{m} e^{-\alpha\left(T-t_{k}\right)} \sqrt{t_{k}-t_{k-1}}\left(Z_{k} \sum_{i=1}^{n} e^{\alpha\left(T-T_{i}\right)} \Delta_{i}\right. \\
\left.-\sum_{i=1}^{n} e^{\alpha\left(T-T_{i}\right)}\right)
\end{gathered}
$$

Combining equations (3.31) and (3.) gives the desired result.

Using the Nigerian interbank offered rate monthly data between January 2007 and July 2020 obtained from the website of Central Bank of Nigeria, we estimated the parameter values as $\alpha=96.2777$ which stands for speed of mean reversion; $\rho=0.1317$ implies that the longterm mean rate; $\sigma=0.7168$ which implies that the volatility of the interest rate is 0.7168 , while the initial interest rate is 0.1317 . Hence, the dynamics of the interbank interest rate considering equation (3.1) becomes

$$
d r_{t}=\alpha\left(\rho-r_{t}\right) d t+\sigma d W_{t}+d J_{t}
$$

The greeks become

$$
=96.27\left(0.1317-r_{t}\right) d t+0.7168 d W_{t}+d J_{t} .
$$

$$
\text { delta }=\Delta=e^{-0.131 T}\left(-T E\left[\Phi\left(r_{T}\right)\right]+E\left[\Phi\left(r_{T}\right) H\left(r_{T}, B_{r_{0}}\right)\right]\right),
$$

where its Malliavin weight in equation (3.26) is given by

$$
H\left(r_{T}, B_{r_{0}}\right)=\frac{e^{-96.277 \pi}}{0.7168} \sum_{k=1}^{m} \frac{e^{96.28\left(T-t_{k}\right)}}{\sqrt{t_{k}-t_{k-1}}} Z_{k}+e^{-96.28 T} \sum_{i=1}^{n} e^{96.28\left(T-T_{i}\right)} \Delta_{i} ;
$$

and from equations (3.27) and (3.30), respectively, we have

$$
\text { vega }=\vartheta=e^{-r_{0} T} E\left[\Phi\left(r_{T}\right) H\left(r_{T}, B_{\sigma}\right)\right]
$$

where

$$
\begin{aligned}
& H\left(r_{T}, B_{\sigma}\right) \\
& =\frac{1}{0.7168} \sum_{k=1}^{m}\left(Z_{k}-1\right) \\
& +\sum_{k=1}^{m} e^{-96.28\left(T-t_{k}\right)} \sqrt{t_{k}-t_{k-1}}\left(Z_{k} \sum_{i=1}^{n} e^{96.28\left(T-T_{i}\right)} \Delta_{i}-\sum_{i=1}^{n} e^{96.28\left(T-T_{i}\right)}\right) .
\end{aligned}
$$

The value of the greek 'delta' lies between -1 and 1 after computation. A delta of 0.4 implies that the interest rate will rise by \#0.40 if the underlying interest rate rises by \#1. The greek 'vega' determines the worth of the interest rate whenever there is $1 \%$ move in its volatility. Hence, the greeks help in monitoring derivatives in order to minimize risks.

\section{Conclusion}

Having a good interest rate model is essential for any economy since interest rates serve as basis for many transactions and decisions. The need to inculcate jumps in a model cannot be overemphasized. In this paper, we have discussed a modified version of the existing Vasicek model to include jumps which is inherent in interest rate paths. We also derived the greeks 'delta' and 'vega' that measure the sensitivities of the interest rates to changes in the initial interest rate and volatility, respectively. These greeks are very useful in minimizing risks.

\section{Declaration of Conflict of Interests}

The authors declare no conflict of interests

\author{
Authors Contribution \\ Conception: [A.M.U, E.O.O, L.S.A, M.N.A] \\ Design: [A.M.U, E.O.O, L.S.A, M.N.A] \\ Execution: [A.M.U, E.O.O.] \\ Interpretation: [A.M.U, E.O.O, L.S.A, M.N.A] \\ Writing the paper: [A.M.U, L.S.A]
}

\section{References}

Bates, D.S., 1996, Jumps and stochastic volatility: Exchange rate processes implicit in Deutsche Mark options. The Review of Financial Studies, 9(1): 69-107.

Bavouzet-Morel, M-P., Messaoud, M, 2006, Computation of greeks using Malliavin calculus in jump-type market models. Electronic Journal of Probability, 10: 276-300.

Berhane, T., Adam, M., Haile, E., 2019, Option pricing on commodity prices using jump diffusion models. International Journal of Mathematical Modelling \& Computations, 9(1): 17-37.

Buraschi, A., Jiltsov, A., 2007, Habit formulation and macroeconomic models of the term structure of interest rates. Journal of Finance, 62(6): 3009-3063.

Carr, P., Mayo, A., 2007, On the numerical evaluation of option prices in jump- diffusion processes. European Journal of Finance, 13(4): 353 372.

Chang, Y., Wang, Y., 2020, Option pricing under double stochastic volatility model with stochastic interest rates and double exponential jumps with stochastic intensity. Mathematical Problems in Engineering, 2020, Article ID 2743676, 13 pages. https://doi.org/10.1155/2020/2743676

Cont, R., Tankov, P., 2004, Financial modelling with jump processes. Chapman \& Hall/ CRC Financial Mathematics Series.Chapman \& Hall/CRC, BocaRaton, FL.

Feng, L., Linetsky, V., 2008, Pricing options in jump-diffusion models: An extrapolation approach. Operations Research, 56(2): 304-325.

Guo, Z-Y., 2021, Bias-correlated estimators for the Vasicek model: An application in risk measure estimation. Journal of Risk, 23(2): 71-104. https://doi:10.21314/JOR.2020.445.

Jiahui, Y., Shengwu, Z., Haitao, Z., Kaiqiang, G., 2019, Pricing vulnerable 
option under jump-diffusion model with incomplete information. Discrete Mathematics in Nature and Society, Article ID 5848375, 8 pages.

Kou, S. G., 2002, A jump-diffusion model for option pricing. Management Science, 48(8): 1086-1101.

Kou, S. G., 2007, Jump-diffusion models for asset pricing in Financial Engineering. In: Birge, J. R. and Linetsky, V., editors. Financial Engineering, vol. 15 of Handbooks in Operations Research and Management Science.Elsevier, 73-116.

Lau, K. J., Goh, Y. K., Lai, A. C., 2019, An empirical study on asymmetric jump-diffusion for option and annuity pricing. PLoS ONE, 14(5): e0216529. https://doi.org/10.1371/journal.pone.0216529.

Merton, R. C., 1976, Option pricing when underlying stock returns are discontinuous. Journal of Financial Economics, 3: 125-144.

Novat, K., Charles, W. M., Masanja, V. G., 2019, Merton's jump diffusion model: An application to stock markets of east African countries. Advances in Scientific Research \& Engineering, 5(8): 15-24.

Perelló, J., Montero, M., Masoliver, J., Farmer, J. D., Geanakoplos, J., 2020, Statistical analysis and stochastic interest rate modelling for valuing the future with implications in climate change mitigation. Journal of Statistical Mechanics: Theory and Experiment, 043210. https://doi.org/10.1088/1742-5468/ab7a1e.
Rao, B. L.S., 2021, Maximum likelihood estimation in the mixed fractional Vasicek model. Journal of the Indian Society for Probability and Statistics. https://doi.org/10.1007/s41096-020-00094-8.

Salmi, S., Toivanen, J., 2011, An Iterative method for pricing American options under jump-diffusion models. Applied Numerical Mathematics, 61(7); 821-831.

Vasicek, O. A., 1977, An equilibrium characterization of the term structure. Journal of Financial Economics, 5: 177-188.

Verschuren, R. M., 2020, Stochastic interest rate modelling using a single or multiple curves: An empirical performance analysis of the Lévy forward price model. Quantitative Finance, 20(7): 1123-1148. https: 10.1080/14697688.2020.1722318

Wang, G., Wang, X., Liu, Z., 2017, Pricing vulnerable American put options under jump-diffusion processes. Probabilities in the Engineering \& Informational Sciences, 31(2): 121-138.

Wu, Y., Liang, X., 2018, Vasicek model with mixed-exponential jumps and its applications in finance and insurance. Advances in Difference Equations, 2018:138. 15pages. https://doi.org/10.1186/s13662018-1593-Z. 